\title{
SOCIETY, LITERATURE AND WOMEN
}

\section{समाज, साहित्य और नारी}

\author{
Dr. Usha Kumari. K.P. 1 四 \\ ${ }^{1}$ Associate Professor, Department of Hindi, Mahatma Gandhi College, Thiruvananthapuram, Kerala, \\ India
}

DOI: https://doi.org/10.29121/granthaalayah.v9.i4.2021.3903

Article Type: Research Article

Article Citation: Dr. Usha Kumari. K.P.. (2021). SOCIETY, LITERATURE AND WOMEN. International Journal of Research GRANTHAALAYAH, 9(4), 615-618. https://doi.org/10.29121/granthaalayah.v9.i4.2021.3903

Received Date: 15 April 2021

Accepted Date: 30 April 2021

\begin{abstract}
English : A society maybe defined as a network of interconnected major groups viewed as a unit and sharing a common culture. It is a group of individuals involved in persistent social interaction. Literature is an imitation of human action, often presents a picture of what people react in the society. What writers of literature do is to convey the real life events in their society through fiction. " Literature is a mirror through which people look at it and absorbs all happenings in the society". After all society is the bond of fellowship between communication that the poet or writer seeks. Women are now getting power in all areas of societies. Education has made women independent. Women are the essential origin of life. A woman is a dawn of light where all darkness disappears. Women with their freshness of their idioms and the newness of their poetics, they have created a significant space for themselves in the literature world. A woman plays a key role in socio-economic development of the society and also strengthens the foundation of family, embracing everyone with the unconditional love.
\end{abstract}

Hindi : एक समाज को परस्पर जुड़े प्रमुखों के नेटवर्क के रूप में परिभाषित किया जा सकता है समूहों को एक इकाई के रूप में देखा जाता है और एक साझा संस्कृति साझा की जाती है। यह एक समूह है लगातार सामाजिक संपर्क में शामिल व्यक्तियों की। साहित्य एक है मानव क्रिया की नकल, अक्सर लोगों की एक तस्वीर प्रस्तुत करती है समाज में प्रतिक्रिया। साहित्य के लेखक क्या करते हैं यह बताने के लिए है कल्पना के माध्यम से उनके समाज में वास्तविक जीवन की घटनाएं। "साहित्य एक दर्पण है" जिसके माध्यम से लोग इसे देखते हैं और सभी घटनाओं को अवशोषित करते हैं समाज"। आखिर समाज आपसी भाईचारा का बंधन है संचार जो कवि या लेखक चाहता है। महिलाओं को अब समाज के सभी क्षेत्रों में सत्ता मिल रही है। शिक्षा महिलाओं को आत्मनिर्भर बनाया है। महिलाएं की आवश्यक उत्पत्ति हैं जिंदगी। नारी प्रकाश का भोर है, जहां सारा अंधकार मिट जाता है। महिलाएं अपने मुहावरों की ताजगी और अपने नएपन से कविताओं में, उन्होंने अपने लिए एक महत्वपूर्ण स्थान बनाया है साहित्य की दुनिया। एक महिला सामाजिक-आर्थिक में महत्वपूर्ण भूमिका निभाती है समाज के विकास और की नींव को भी मजबूत करता है परिवार, बिना शर्त प्यार के सभी को गले लगाता है।

Keywords: महिला सशक्तिकरण; समाज और साहित्य; महिला और साहित्य

(c) 2021 The Author(s). This is an open access article distributed under the terms of the Creative Commons Attribution License, which permits unrestricted use, distribution, and reproduction in any medium, provided the original author and source are credited. 
डॉ उषा कुमारी के.पी.

\section{1. प्रस्तावना}

व्यक्ति, समाज और साहित्य के परस्पर सम्बन्ध को जानते हुए हम नारी के विविध रूप को पहचान सकते हैं। नर-नारी के पारस्परिक सम्बन्ध से सृष्टि का विकास होता है। दोनों का व्यक्तित्व विविध रूप ग्रहण कर लेता है। पुरुष नारी की अपेक्षा प्रकृति की ओर से भी अधिक स्वाधीन है और समाज की ओर से भी, जबकि नारी प्रकृति और समाज दोनों की ओर से पुरूष पर निर्भर है। आधुनिक युग में नारी की निर्भरता, आर्थिक एवं शैक्षणिक स्वावलम्बन से कम हुई है, किन्तु स्थिति लगभग वैसी ही है। डॉ. श्यामचरण दुबे का मत है- "प्रत्येक देश की संस्कृति में एक ओर मानव और प्रकृति के पारस्परिक सम्बन्ध है, तो दूसरी ओर मानवीय धरातल पर सम्बन्धों का संगठन अनिवार्य भी होता है और तीसरी ओर अटश्य जगत् की उन शक्तियों से भी समझौता करना पड़ता है, जिनके सम्बन्ध में कार्य कारण की बुद्धि अधिक सहायता नहीं करती।“

व्यक्ति और समाज के बीच द्वन्द्ध ही विकास और विघटन का मूल कारण है। व्यक्ति और समाज के बीच महत्वपूर्ण ईकाई परिवार है। परिवार नर-नारी को संरक्षण और सुरक्षा प्रदान करता है। परिवार की परिभाषा जुकरमैन के अनुसार - "एक परिवार-समूह पुरूष स्वामी, उसकी स्त्री या स्त्रियों और उनके बच्चों से मिलवार बनता है और कभी उसमें एक या अधिक अविवाहित पुरूष भी सम्मिलित रहते है।“‘

प्रत्येक समाज में नारी की स्थिति उस समाज में प्रचलित मान्यताओं, आदर्शों, मूल्यों के अनुसार निश्चित होती है। समाज की अधिकांश मान्यताऐं, पुरूष द्वारा निर्मित होने के कारण नारी के स्वतन्त्र अस्तित्व को स्वीकार नहीं किया गया। नारी जैविक रूप से मादा है। व्यक्तित्व तथा प्रकृतितत्व गुणों के आधार पर उसे नारी के रूप में प्रतिष्ठित किया जाता है। इन दोनों रूपों में नारी पुरूष से भिन्न है, लेकिन उसकी तीसरी स्थिति जो उसे ‘मानवी‘ रूप में प्रतिष्ठित करती है, उसके अन्तर्गत ही वे सभी रूप समाहित होते हैं।

आशारानी ब्योरा के अनुसार - "नारी, नारी होकर भी मातृत्व और पत्नीत्व की भूमिका निभाते हुए भी, सर्वप्रथम मनुष्य है, मानवी है। मानवता के अधिकार और दायित्व को लेकर चलने से ही वह अपनी हीनताजन्य कुठांओं से बच सकेगी और नयी पीढ़ी को बेहत्तर मानवीय संस्कारों से समृद्ध कर सकेगी।“"

आज की नारी के लिए पारिवारिक सम्बन्ध मूलाधार है। स्वातन्त्रोत्तर भारत में नारी के विविध रूपों को प्राचीन और नवीन मूल्यों, परम्परागत और परिवर्तित संवेदनाओं, अनुभूतियों एवं प्रवृत्तियों के माध्यम से अभिव्यक्ति ही है जिसमें नारी के विविध रूपों में विकास विघटन और बदलाव के बिन्दु परिलक्षित होते हैं।

परिवार में नारी का कन्या, पत्नी, माँ और बहन रूप से स्वतः सम्बन्ध जुड़ जाता है, क्योंकि उसके व्यक्तित्व के विकास से इसका गहरा सम्बन्ध है। नारी के अन्य रूप, ननंद, देवरानी, जेठानी, और भाभी आदि गौड़ महत्व रखते है। नारी का विधवा रूप भी चर्चित है। नारी का प्रेमिका के रूप में पारिवारिक एवं सामाजिक हृष्टि से मान्य नहीं रखता, क्योंकि प्रेमिका रूप परिवार एवं समाज को मान्य नहीं है। स्त्री अपनी संस्कारों से बंधती है।

उत्रीसवी सदी में महिलाओं का सुधार आरम्भ हुआ। समाज सेवी सुधारकों ने नारी उत्थान का जयघोष किया। आज भी नारी सम्बन्धी नियम में कोई बहुत बड़ा परिवर्तन नहीं आया है। लेकिन आज नारी शिक्षित होने के कारण वह अपनी स्थिति के प्रति अस्वस्थ हो उठी है। नारी की आर्थिक स्वतन्त्रता उसे आज परिवार की आर्थिक स्थिति सुधारने, समय और शिक्षा के उचित उपयोग, अपने व्यक्तित्व के विकास, कैरियर की इच्छा, स्वतन्त्र आय की प्राप्ति आदि प्राप्त करने लायक बना रही है। एक "व्यक्तित्व सम्पन्न मनुष्य “ रूप में नारी की स्थापना हुई है।

धर के बाहर विभित्र क्षेत्रों में काम करने वाली नारी में आत्मविश्वास का उदय हुआ है, पति और परिवार से आगे बढ़कर उसने अपने को समाज का एक उपयोगी, महत्वपूर्ण अंग माना है, लेकिन आज वह समाज और परिवार में स्वतन्त्र रूप से जी रही है।

आधुनिक नारी की सबसे महत्वपूर्ण विशेषता यह है कि वह आत्मनिर्भर दिखाई देती है। आज नारी में सामाजिक, आर्थिक, धार्मिक, सांस्कृतिक चेतना भी दिखाई देती है। नारी का सोचना-समझना, सावधान होना, एवं सर्वकता से व्यवहार करना ही नारी चेतना माना जा सकता है।

हिन्दी साहित्य समय और समाज के सांगोपांग चित्रित करने के लिए सबसे प्रभावशाली विधा है। वह मानव-जीवन की यथार्थता, सत्यता, आवश्यककताएं, संभावनाएं, समस्याएं, मूल्यों के उत्थान-पतन आदि का निरूपण उत्पन्न होने वाले वांछित और अवांछित परिवर्तनों को प्रतिबिबिंत करनेवाले दर्पण के रूप में साहित्य का महत्व सर्वाधिक सिद्ध हो गया है। 
समाज तेजी से बदल रहा है। विश्वास का एक नया घेरा नारियों के बीच अपना स्थान बना रहा है। भारतीय संस्कृति, समाज, धर्म, अर्थ, राजनीति आदि परिवेशों से और घटनाओं से, समस्याओं से प्रभावित होकर नारी लेखन ने यथार्थता एवं कल्पना तत्व का समन्वय करने की कोशिश की है।

समकालीन लेखन में महिलाओं ने अपना कामान इस तरह संभाली है जिस तरह वे अन्य गति विधियों में मोर्चा संभालती है। हाशिये और कोष्टक से निकल कर केन्द्र में आने के लिए पिछली पीढ़ी की लेखिकाओं ने अथक परिश्रम किया। आज महिला लेखन विश्वविधालय का एक महत्वपूर्ण विषय है।

आधुनिक कहानी कला के विकास में महिला-कथाकारों ने नारी की विविध रूप, शक्ति, समस्या, एवं चेतना पर अधिक ध्यान केन्द्रित करके अपनी कहानियों में मार्मिक चित्रण किया है। शिल्प की दृष्टि से भी कलात्मकता के दर्शन होते हैं।

उन्होंने अपनी कहानियों में संघर्षमय तनावपूर्ण शहरी जीवन में सांस ले रहे परिवारों के टूटने तथा व्यक्ति के टूटने की कथा कही है। वर्तमान नारी की उभरती हुई परिवर्तन एवं विकासशील मानसिकता तथा उसके अहं का चित्रण भी बड़ी खुबी के साथ प्रस्तुत किया है। कहानी सुनाने से लेकर आधुनिक कहानी कला के विकास में महिला कहानिकारों ने नारी के विविध रूप, शक्ति, समस्या एवं चेतना पर ध्यान केन्द्रित किया।

हिन्दी उपन्यासों में महिला कथाकारों का प्रवेश अधिक जोरों से हुआ। वे उपन्यास लेखन में इतनी सक्रिय थी कि अब सारा उपन्यास कथा क्षेत्र नारियों के हाथ में है। स्त्री लेखन में मौजूद खास संवेदना की वजह से इसे आजकल विशेष दर्जा दिया गया है। स्त्री लेखन के संदर्भ में आर. शशि धरन् ने लिखा है, महिला लेखन अपनी तमाम खूबियों और खामियों के बावजूद आधी दुनिया को अपनी रचनाओं में बड़ी खूबसूरती के साथ उपस्थित कर गया है और कर रहा है।

लेखिकाओं ने महज नारी जीवन पर ही नहीं लिखा है बल्कि जीवन की तमाम संवेदनाओं की अभूतपूर्व निकासी कर के कई मायनों में पुरूषों से भी ज्यादा कामयाबी हासिल की है। कलम से नारी के विषय में जो कुछ लिखा गया है वह अत्यन्त सार्थक और सामाजिक है। महिला लेखन परिव्याप्ति के खुलने और जीवन की तस्वीर के पूरा हो जाने का साक्ष्य है।

लेखिका अब साहित्य की हर विधा के द्वारा अपने-आपको अभिव्यक्त कर रही है। वह साहित्य की सभी विधाओं में लेखन कर रही है। इसकी ही एक सशक्त झलक, साठ के बाद का नारी विमर्श है। हिन्दी गद्यत्तर साहित्य में आत्मकथा साहित्य का स्थान अत्यंत महत्वपूर्ण है। आत्मकथा साहित्य में स्वयं जीवन के अतीत को अभिव्यक्त करता है। स्त्री अपने आत्मकथाओं के माध्यम से अपने व्यक्तिगत जीवन या इसी समाज में घटित घटना के माध्यम से नारी के यातनामय जीवन को भी प्रस्तुत करती है।

कविता के क्षेत्र में भी नारी पीधे नहीं है। “अपमान मत करना नारियों का, इनके बल पर जग चलता है, पुरुष जन्म लेकर तो .... इन्ही की गोद में पलता है। कोई भी देश तरक्की के शिखर पर तब तक नहीं पहुँच सकता, जब तक उसकी महिलाएं कंधे से कन्धा मिला कर ना चले। नारी का सोचना, समझना, और सावधान होना एवं सर्वकता के व्यवहार करना ही नारी चेतना माना जा सकता है।

हिन्दी साहित्य में महिला लेखन ने नई दिशा एवं नया मोड़ देकर अभूतपूर्व प्रेरणास्पद, प्रशंसनीय योगदान दिया है। नारी अधिक ईमानदार, निष्ठावान, कर्मठ, धर्मवान और बलिदान करने को सदा तत्पर रहनेवाली एक ऐसी जीव है जैसा दुनिया में और कोई नहीं हो सकता।

"क्यों कहती है दुनिया कि नारी कमजोर हैं, आज भी नारी के हाथों में घर चलाने की डोर है दुनिया की पहचान है औरत, घर-घर की शान है औरत है।

\section{SOURCES OF FUNDING}

None.

\section{CONFLICT OF INTEREST}

None. 


\section{डॉ उषा कुमारी के.पी.}

\section{ACKNOWLEDGMENT}

None.

\section{REFERENCES}

[1] Aurath Ke Liye Aurath - Nasira Sharma.

[2] The Social Life of Moneys End- S Jukerman.

[3] Bharatiye Naari- Asharani Bohra. 